\title{
Antibiotic Prophylaxis in Orthopedic Surgeries: The Results of an Implemented Protocol
}

\author{
Raquel Queiroz $^{1}$, Renato S. Grinbaum ${ }^{1}$, \\ Luciana L. Galvão ${ }^{1}$, Fernando G.Tavares ${ }^{1}$ \\ and Gun Bergsten-Mendes ${ }^{2}$
}

\author{
${ }^{1}$ Hospital of Servidor Público Estadual; São Paulo, SP; \\ ${ }^{2}$ Department of Pharmacology, Faculty of Medical Sciences, \\ State University of Campinas; Campinas, SP, Brazil
}

\begin{abstract}
Though the basic principles of antibiotic prophylaxis have been well established, there is still considerable incorrect usage, including how much is prescribed and especially in the duration of treatment, which is generally superior to what is indicated. The adequate use of these drugs contributes towards decreasing the time of internment of the patient, prevents surgical site infection (SSI), decreasing the development of resistant microorganisms, and towards reduced costs for the hospital pharmacy. A protocol for the use of antibiotic prophylaxis in the Orthopedics and Traumatology Service of the Hospital do Servidor Público Estadual de São Paulo was developed. The objectives of the study were to promote rational antibiotic surgical prophylaxis, through the implantation of a protocol for the use of these drugs in a surgical unit, with the direct contribution of a druggist in collaboration with the Infection Control Committee, to evaluate the adhesion of the health team to the protocol during three distinct periods (daily pre-protocol, early post-protocol and late post-protocol) and to define the consumption of antimicrobials used, measured as daily defined dose.

Key Words: Antibiotic prophylaxis, protocol, surgical site infection, pharmacy.
\end{abstract}

The prophylactic use of antibiotics is but one of many measures to reduce the risk of surgical site infection (SSI), the most common nosocomial infections among surgical patients [1]. Though a lowered incidence of SSI with the use of prophylactic antibiotics is well documented, the inappropriateness of antibiotic prophylaxis has also been extensively demonstrated in many situations. Antibiotics are often used in wrong doses, for too long, and with too broad a spectrum of antimicrobial activity [2-5]. Antimicrobial resistance, superinfection and unnecessary costs are common consequences of inappropriate surgical antibiotic prophylaxis $[3,6]$.

Received on 27 January 2005; revised 11 June 2005.

Address for correspondence: Dr. Raquel Queiroz de Araújo. Farmácia Hospitalar do Servidor Público Estadual/HSPE. Rua Pedro de Toldo, $1800,1^{\circ}$ andar, bloco F. Zip code: 04039-901.

São Paulo, SP, Brazil. E-mail: raquelq@uol.com.br

The Brazilian Journal of Infectious Diseases 2005;9(3):283-287 (C) 2005 by The Brazilian Journal of Infectious Diseases and Contexto Publishing. All rights reserved.
Many multidisciplinary educational and managerial interventions have been implemented in attempts to modify this scenario [7-9].

The use of antibiotics as prophylaxis for SSI in orthopedic surgeries is indicated when prostheses are implanted, or when any sort of osteosynthetic materials, such as nails, wires, plates, and screws, are used. In these cases cefazolin, a first generation cephalosporin, is the antibiotic of choice [1,10-13].

We describe a multidisciplinary experience in implanting a protocol for prophylactic antibiotic prescription in the orthopedic department of a general hospital.

\section{Material and Methods}

\section{$\underline{\text { Hospital }}$}

The study was conducted in a 711-bed general public hospital. The orthopedic ward had 37 beds and performed an average of 87 surgeries per month 
during 1999. There were no formal guidelines for surgical antibiotic prophylaxis in the orthopedic surgeries.

\section{$\underline{\text { Protocol }}$}

The protocol for surgical antibiotic prophylaxis was prepared at the request of the head of the orthopedic department; it was the consensus result of a multidisciplinary discussion group involving the medical staff, members of the Infection Control Committee (ICC) and the pharmacist. This protocol complied with internationally-accepted guidelines [14-16]. To promote compliance with the protocol, the multidisciplinary team offered lectures on the subject to the residents, and discussed the rationale of perioperative antibiotic prophylaxis during the daily ward visits of the medical staff.

\section{The role of the hospital pharmacy}

The hospital pharmacy developed a form for dispensing prophylactic antibiotics based on the protocol. The surgeon signed the antibiotic order form the day before surgery for elective surgeries, and the pharmacist assured that the patients received the correct intra - and postoperative doses of the indicated antibiotic in the operating room. The pharmacist also provided emergency antibiotic kits whenever needed. Each time a prophylactic antibiotic prescription that was not in accordance with the protocol reached the pharmacy, the pharmacist asked the prescriber the reason for the prescription. If the prescriber insisted on the prescription, a physician from the ICC was called in to discuss the issue until a consensus decision was reached. This decision involved either the withdrawal of the antibiotic or modification of the antibiotic schedule.

\section{$\underline{\text { Data collection }}$}

The data were collected by the pharmacist from the medical records, from the daily prescription sheets, and during the daily medical ward visits. Prescriptions that diverged from the protocol were considered "errors". Six aspects concerning antibiotic prophylaxis were evaluated; they included the indication of antibiotic prophylaxis, the choice of the antibiotic, the timing of the first prophylactic dose, the prescribed dose, the need for extra intraoperative doses when indicated, and the number of postoperative doses. The SSI rates during the three study periods (see below) were recorded.

The amount of prophylactic antibiotics prescribed during the study periods was expressed in daily defined doses (DDD)/100 bed-days [17,18].

\section{$\underline{\text { Study design }}$}

All surgical orthopedic patients not on antibiotic treatment were included in the study. Three study periods were designed. The first, from July to September 1998, described the pre-protocol pattern of prophylactic antibiotic prescriptions. During this three-month period, the multidisciplinary team established close contact with the staff of the orthopedic department. The prophylaxis protocol started on September 1998, and a three-month period was allowed to consolidate the new praxis. An early postprotocol survey was conducted from December 1998 to February 1999. From then on, the multidisciplinary team only went to the orthopedic ward when requested, on average once a week. A third late postprotocol survey was carried out five months later, from July to September 1999, in order to evaluate the compliance with the established protocol after a period without the daily presence of the pharmacist and the ICC representatives.

\section{$\underline{\text { Exclusion criteria }}$}

Surgical orthopedic patients were excluded from the study if: 1) they had been on therapeutic antibiotics before the surgery, 2) the postoperative follow up was missed, for example, by discharge within $24 \mathrm{~h}$ of surgery, 3 ) the patient needed a further surgery within 72 hours, or 4) information about the intraoperative use of antibiotics was lacking. 


\section{Costevaluation}

The cost of the prophylactic antibiotics was expressed as cost/surgery and as the cost of the DDD of prophylactic antibiotics in each study period. The values were expressed in US\$.

\section{$\underline{\text { Statistical analysis }}$}

The chi-square test and Fisher's exact test were used as appropriate. Statistical significance was considered when $\mathrm{p} \leq 0.05$. The statistical analysis was done using the software EpiInfo, version 6.1 (CDC, Atlanta, GA).

\section{Results}

$\underline{\text { Patients and surgeries }}$

Of the 299 surgeries evaluated, 74 were in the preprotocol period, 113 were in the early post-protocol period and 112 were in the late post-protocol period. Nine patients met the exclusion criteria. Forty percent of the patients were male, and the mean age of all the patients was $52.5 \pm 25.7$ years. There were no significant differences in the age or gender composition of the patients in the three study periods.

Sixty-eight percent of the surgeries were for osteosyntheses and total hip arthroplasties. More than $95 \%$ of the surgeries were elective and clean. There was no significant difference in the rate of incidence of SSI in the three study periods ( $4.0 \%$ in the pre-protocol, $3.6 \%$ in the early post-protocol, and $2.8 \%$ in the late post protocol periods). The number of "errors" in the three study periods and the respective incidence of SSI were not significantly related $\left(\chi^{2}=2.52, \mathrm{p}=0.28\right)$.

\section{$\underline{\text { Surgical antibiotic prophylaxis }}$}

Prophylactic antibiotics were prescribed in 237 surgeries $(81.7 \%)$. The DDD/100 bed-days for the prophylactic antibiotic in the pre-protocol period (cefalotin) was 8.53 , and in early post-protocol and in late post-protocol periods (cefazolin) it was 9.42 and 7.90 , respectively.

The frequency of the appropriateness of the antibiotic prophylaxis according to the established protocol during the three study periods was determined (Table 1). The number of "errors" was analyzed by recording the number of times there were prescriptions that disagreed with the established protocol for each patient. In the pre-protocol period, only $3.3 \%$ of the surgeries with antibiotic prophylaxis had no "errors", but this value reached 50\% in the late post-protocol period ( $\mathrm{p}<$ .0001) (Figure 1).

\section{Cost of the antibiotic prophylaxis}

Cost per surgery. For the 61 surgeries in the preprotocol period, the expenditure for antibiotics was US $\$ 16.62 /$ surgery. For 176 surgeries with antibiotic prophylaxis in the two post-protocol periods, the expenditure fell to US $\$ 6.61 /$ surgery.

Cost of the DDD. In the pre-protocol period, 8.53 $\mathrm{DDD} / 100$ beds/day of cefalotin cost US $\$ 36.49$. In the early post-protocol period, $9.42 \mathrm{DDD} / 100$ beds/day of cefazolin cost US $\$ 29.13$, and in the late postprotocol period, $7.90 \mathrm{DDD} / 100$ beds/day of cefazolin cost US\$24.43.

\section{Discussion}

The improvement in the pattern of surgical antibiotic prophylaxis resulted from the joint efforts of a multidisciplinary team, which provided educational intervention, and the hospital pharmacy, which made managerial adjustments to dispense prophylactic antibiotics according to the protocol and to check compliance with the established protocol. Educational plus restrictive interventions gave the best results for rational antibiotic prophylaxis [19].

The frequency of the proper indication of prophylactic antibiotics in orthopedic surgeries was not affected by the protocol, probably because most of the surgeries performed in this orthopedic department 
Table 1. Frequency $(\%)$ of the appropriateness of antibiotic prophylaxis in orthopedic surgeries during three study periods

\begin{tabular}{lccrr}
\hline Items & \multicolumn{3}{c}{ Study period } & P \\
\cline { 2 - 5 } & Pre-protocol & Early post-protocol & Late post-protocol \\
\hline Indication of antibiotic prophylaxis & 84.8 & 94.5 & 91.6 & $\mathrm{NS}$ \\
Choice of antibiotic & 98.3 & 100.0 & 100.0 & $\mathrm{NS}$ \\
Timing of the first prophylactic dose & 80.0 & 94.9 & 95.6 & $<.05$ \\
Prescribed dose & 38.3 & 74.4 & 80.9 & $<.05$ \\
Extra intraoperative doses & 10.2 & 69.2 & 80.9 & $<.05$ \\
Number of postoperative doses & 33.3 & 83.3 & 83.8 & $<.05$ \\
\hline
\end{tabular}

$\mathrm{NS}=$ not significant

Figure 1. Distribution of the number of "errors" relative to the established antibiotic prophylaxis protocol during the three study periods.

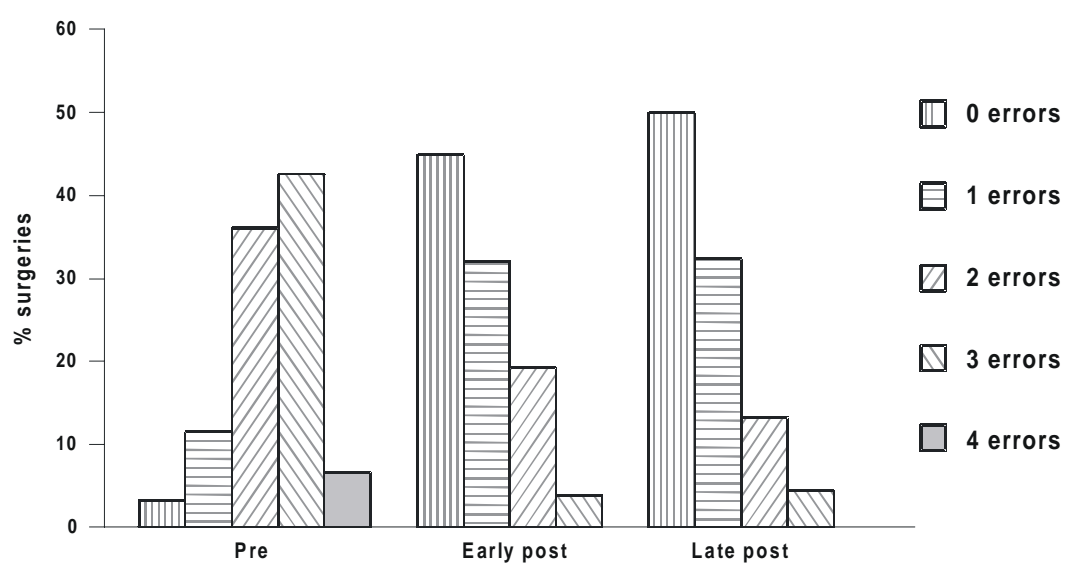

used osteosynthetic materials or implant prostheses, and in both situations antibiotic prophylaxis is indicated. The same observation applies to the choice of antibiotic. For orthopedic surgeries, first generation cefalosporins are recommended, and before the protocol the prescribed antibiotic was cefalotin. Despite the wellknown pharmacokinetic advantages of cefazolin $[1,10,20]$, which was recommended in the established protocol, the use of cefalotin in the pre-protocol period cannot be considered incorrect.
There were significant differences in the prophylactic antibiotic prescriptions after the implantation of the protocol, particularly in the dosages, the intraoperative repetition of the antibiotic, and the number of postoperative doses. Excessive duration of antibiotic prophylaxis is one of the most common errors in surgical antibiotic prophylaxis [3]. It is noteworthy that compliance with the protocol remained high months after the enforcing multidisciplinary team stopped its daily visits to the orthopedic ward. Apart from the 
probable clinical benefits for the patients, there was marked reduction in the cost of the antibiotic prophylaxis, thus strengthening the need for enforcing the proper use of antibiotics in the prophylaxis of SSI. Since the costs of the two antibiotics for the hospital were almost the same, the reduction in costs resulted mainly from the proper number of postoperative doses.

\section{References}

1. Gyssens I.C. Preventing postoperative infections: current treatment recommendations. Drugs 1999;57:175-85.

2. Currier J.S., Campbell H., Platt R., Kaiser A.B. Perioperative antimicrobial prophylaxis in middle Tennessee. Rev Infect Dis, 1991;13 (Suppl 10):S874-S8.

3. Gorecki P., Schein M., Rucinski J.C., Wise L. Antibiotic administration in patients undergoing common surgical procedures in a community teaching hospital: the chaos continues. World J Surg 1999;23:429-33.

4. Holzeheimer R.G., Haupt W., Thiede A., Schwarzkopf A. The challenge of postoperative infections: does the surgeon make a difference? Infect Control Hosp Epidemiol 1997; 18:449-56.

5. Mini E., Nobili S., Periti P. Methicillin-resistant staphylococci in clean surgery is there a role for prophylaxis? Drugs 1997;54(Suppl 6):39-52.

6. Mcgowan Jr. J.E. Cost and benefit of perioperative antimicrobial prophylaxis: methods for economic analysis. Rev Infect Dis 1991;13(Suppl 10):S879-S89.

7. Codina C., Trilla A., Riera N., et al. Perioperative antibiotic prophylaxis in Spanish hospitals: results of a questionnaire survey. Infect Control Hosp Epidemiol, 1999; 20:436-9.

8. Jonston J., Harris J., Hall J.C. The effect of and educational intervention on the use of perioperative antimicrobial agents. Aust Clin Rev 1992;12:53-6.

9. Martin C., Pourriat J.L. Quality of perioperative antibiotic administration by French anaesthesists. J Hosp Infect 1998; 40:47-53.

10. Anonymous. Antimicrobial prophylaxis in surgery. Med Lett, 1999;41:75-80.

11. Court-Brown C.M. Antibiotic prophylaxis in orthopaedic surgery. Scand J Infect Dis 1999;(Suppl 70):74-9.

12. Oishi C.S., Carrion W.V., Hoaglund F.T. Use of parenteral prophylactic antibiotics in clean orthopaedic surgery. A review of the literature. Clin Orthop 1993;296:249-55.

13. Paluzzi R.G. Antimicrobial prophylaxis for surgery. Med Clin North Am 1993;77:427-41.
14. ASHP Commission on therapeutics. ASHP therapeutic guidelines on antimicrobial prophylaxis in surgery. Clin Pharm, 1992;11:483-513.

15. Martin C. Antimicrobial prophylaxis in surgery: general concepts and clinical guidelines. Infect Control Hosp Epidemiol 1994; $15: 463-71$.

16. Page C.P., Bohnen J.M.A., Fletcher J. R., et al. Antimicrobial prophylaxis for surgical wounds: guidelines for clinical care. Arch Surg 1993; $128: 79-88$.

17. Collaborating Centre For Drug Statistics Methodology. ATC index. Including DDDs for plain substances. WHO: Oslo, 1995.

18. Collaborating Centre For Drug Statistics Methodology. Guidelines for ATC classification and DDD assignment. WHO: Oslo, 1995.

19. Fonseca S.N.S., Kunzle S.R.M., Silva S.B., et al. Cost reduction with successful implementation of an antibiotic prophylaxis program in a private hospital in Ribeirão Preto, Brazil. Infect Control Hosp Epidemiol, 1999;20:77-9.

20. Redington J., Ebert S.C., Craig W. Role of antimicrobial pharmacokinetics and pharmacodynamics in surgical prophylaxis. Rev Infect Dis 1991;13(Suppl 10):S790S9. 